\title{
El estudio de caso como alternativa metodológica en la investigación en educación física, deportey actividadfísica. Conceptualización y aplicación \\ Case study as a methodological alternative in research in physical education, sport, and physical activity. Conceptualization and application \\ Beatriz Elena Chaverra Fernández, Didier Fernando Gaviria Cortés, Enoc Valentin González Palacio \\ Universidad deAntioquia (Colombia)
}

Resumen: En este artículo de revisión presentamos el estudio de caso como una opción metodológica para investigar en el campo de la educación física, el deporte y la actividad física. En primer lugar, partimos del vínculo del estudio de caso con la investigación cualitativa, para luego profundizar en sus definiciones, características, clasificaciones, principales críticas y los criterios de rigor y credibilidad necesarios. Finalizamos el texto reseñando algunas investigaciones que se han llevado a cabo en el área en los últimos años con esta metodología; estas investigaciones son una muestra de la diversidad de contextos, participantes y objetivos que pueden ser abordados a través del estudio de caso. En definitiva, pretendemos generar una reflexión en los actores educativos sobre las posibilidades del estudio de caso como una alternativa viable, rigurosa y pertinente que puede orientar sus procesos investigativos y fortalecer el cuerpo de conocimiento de la educación física, el deporte y la actividad física.

Palabras clave: Estudio de caso, educación física, deporte, investigación pedagógica

\begin{abstract}
In this review article we present case study as a methodological option to research in the field of physical education, sport, and physical activity. Firstly, we discuss the relation between case study and qualitative research; successively, we delve into its definitions, characteristics, classifications, main criticisms, and criteria of rigor and credibility. We conclude the work by outlining research that has been carried out in the area in recent years employing this methodology; these studies are an evidence of how diverse contexts, participants, and objectives may be when applying the case study methodology. In brief, we intend to prompt educational actors' reflection about the potentiality of case study as a viable, rigorous, and pertinent alternative that can guide their investigative processes and strengthen the body of knowledge of physical education, sport, and physical activity. Key words: Case studies, physical education, sport, pedagogical research.
\end{abstract}

Resumo: Neste artigo de revisão, apresentamos o estudo de caso como uma alternativa metodológica para a pesquisa no campo da educação física, esporte e atividade física. Primeiro, começamos a partir da ligação do estudo de caso com a pesquisa qualitativa e aprofundamos suas definições, características, classificações, principais críticas e critérios de rigor e credibilidade. Concluímos o texto ao delinear algumas pesquisas realizadas nesta área nos últimos anos com essa metodologia; essas investigações são uma amostra da diversidade de contextos, participantes e objetivos que podem ser abordados através do estudo de caso. Em suma, pretendemos gerar uma reflexão nos atores educacionais sobre as possibilidades do estudo de caso como uma alternativa viável, rigorosa e pertinente que pode orientar seus processos de investigação e fortalecer o conhecimento sobre educação física, esporte e atividade física.

Palavras-chave: estudo de caso, educação física, esporte, pesquisa pedagógica.

\section{Introducción}

Cada vez es más frecuente encontrar investigaciones cualitativas en elárea de educación física, como una manera de acercarse a los fenómenos que allí se presentan, rompiendo con la mirada cuantitativa del movimiento a la cual ha estado ligada históricamente. Es así como los actores del campo, asumen diferentes opciones cualitativas para resolver sus preguntas, y de allí surge el estudio de casos como alternativa metodológica.

En este sentido, Silverman (1987), Silverman y Manson (2003) y Chatoupis y Vagenas (2011), plantean que la mayoría de las investigaciones sobre la enseñanza y en educación física, se han centrado en modelos técnicos y cuantitativos, donde se ha estudiado la comparación de métodos de enseñanza, los comportamientos del profesorado, el tiempo del estudiantado en las tareas y sus actitudes, entre otros aspectos de la educación física, el deporte y la actividad física.

El interés por el cambio de los métodos cuantitativos a los métodos cualitativos, busca modelos de interpretación de los diferentes procesos que se dan en el aula de clase y contribuyen a una mejor comprensión de la interacción entre el profesorado, el alumnado y el conocimiento (Hernández, 2004). En este sentido, López, Monjas y Pérez (2003) afirman que, la investigación desde una racionalidad práctica, está amparada en paradigmas interpretativos, comprensivos y fenomenológicos con los cuales se trata de «iluminar los procesos históricos y sociales que fundamentan el pensamiento y la acción de las personas y grupos, de tal forma que permite la interpretación de las circunstancias sociales y educativas actuales en situaciones reales y concretas» (p. 48).

Fecha recepción: 27-09-17. Fecha de aceptación: 02-10-18 Beatriz Elena Chaverra Fernández

beatriz.chaverra@udea.edu.co
En este artículo realizamos una aproximación conceptual al estudio de caso, sus características básicas, los diferentes tipos, las principales críticas y limitaciones, así como una pequeña selección de investigaciones que han asumido el estudio de caso en la educación física para ejemplificar como se ha llevado a cabo esta metodología en el campo. A partir de nuestra experiencia como investigadores y teniendo como referencia la literatura sobre el tema, pretendemos generar una reflexión en los actores educativos, sobre las condiciones e implicaciones de la utilización del estudio de casos en la investigación en la educación física, la actividad física y el deporte, con el propósito de promoverlo como una alternativa metodológica.

\section{El estudio de caso como parte de la investigación cualitativa}

La investigación cualitativa ha tomado gran fuerza en los estudios educativos, debido a sus aportes para comprender las complejas relaciones que se establecen entre los actores. Como lo plantean diversos autores (Flick, 2004; Galeano, 2004a; Massot, Dorio, \& Sabariego, 2012), la metodología cualitativa permite abordar la realidad subjetiva e intersubjetiva como objetos legítimos de conocimientos científicos, buscando desde la interioridad de los actores, las lógicas de pensamiento que guían sus acciones. En esta misma perspectiva, Taylor y Bogdan (1987) argumentan que, si estudiamos a las personas mediante técnicas cualitativas, llegamos a conocerlas en lo personal y a experimentar lo que ellas sienten en sus luchas cotidianas en la sociedad.

En esta misma línea, Strauss y Corbin (2002) aseguran que la investigación cualitativa permite obtener detalles complejos de algunos fenómenos, como sentimientos, procesos de pensamiento y emociones, los cuales son difíciles de extraer o de aprehender por otros métodos de investigación. Es así, como el proceso cualitativo, rescata la voz de los participantes y sus vivencias, en la búsqueda de construir una descripción e interpretación profunda de aquello que los participantes 
en la investigación afirman o niegan, de lo que hacen y creen hacer, de lo que perciben de su realidad y de su papel en un contexto determinado.

En coherencia, los enfoques cualitativos en la investigación educativa, tienen como tarea principal, la interpretación de la acción social y desvelar su significado (Goetz \& LeCompte, 1988); no la de elaborar teorías científicas que puedan contrastarse de modo experimental (Pérez, 1999). En consecuencia, no podría afirmarse la existencia de una sola forma legítima de hacer investigación cualitativa, ni una única posiciónu orientación común que la sustente (Stake, 2010). En términos de metodologías, perspectivas y estrategias, la investigación cualitativa ha desarrollado diferentes enfoques y orientaciones, en las cuales las distintas tradiciones intelectuales y disciplinarias recrean sus propios métodos y prácticas, cuyos resultados son diversas concepciones acerca de la realidad y cómo conocerla (Rodríguez, Gil, \& García, 1999).

\section{¿Qué es un estudio de caso?}

El diseño de estudio de caso, se inserta en el marco de los estudios cualitativos y la indagación naturalista (Pérez, 2008). Según Yin (1984), el estudio de caso es una forma de investigación con entidad propia, investiga empíricamente un fenómeno contemporáneo dentro de un contexto de la vida cotidiana. Stake (2010) plantea el estudio de casos como algo específico y complejo que se encuentra en funcionamiento y de lo cual se pretende lograr una mayor comprensión y profundizar en aspectos que no son habituales mediante otros diseños.

Según Galeano (2004b), el objetivo del estudio de casos es comprender el significado de una experiencia, e implica el examen intenso y profundo de diversos aspectos de un mismo fenómeno, es decir, es un examen de un fenómeno específico. «El estudio de casos se desarrolla en las situaciones reales en las que los individuos estudiados tienen responsabilidades y obligaciones con las que el estudio puede interferir» (Pérez, 2008, p. 443).

Un caso según Coller (2000), es un objeto de estudio con unas fronteras más o menos claras que se analiza en su contexto real y que se considera relevante, bien sea para comprobar, ilustrar, o construir un conocimiento o una parte de él, o por su valor intrínseco. El caso puede ser una persona, un programa, un evento, un proceso, un grupo social; el cual puede apreciarse en su singularidad y complejidad al insertarse en sus contextos y su interrelación con ellos (Merriam, 1988; Stake, 2010; Yin, 1984). Sánchez (2013) plantea que, algunos puntos para definir el caso están relacionados con los límites físicos o sociales que le confieren a la cosa identidad, es decir, el caso puede ser una escuela concreta, pero también uno o varios de sus profesores, un proyecto curricular o una sola área del mismo, una política educativa del ámbito geográfico al que pertenece la escuela, entre otros.

Los estudios de caso pueden ser muy cuantitativos y poner a prueba la teoría, pero en educación, los investigadores suelen llevarlos a cabo cualitativamente (Merriam, 1988). Algo similar es lo que plantean Cohen, Manion y Morrison (2007), cuando dicen que, el estudio de caso puede comprender situaciones que no siempre son susceptibles de análisis numérico, los contextos son únicos y dinámicos, por tanto, el estudio de caso investiga las complejas interacciones y la evolución de los acontecimientos, las relaciones humanas y otros factores que se presentan en el caso.

Igualmente, Gerring (2004), Martínez (2006) y Stake(2010), reconocen que la investigación a través del estudio de caso, se puede realizar de manera cualitativa o cuantitativa, de forma analítica o integral, por medio de medidas o interpretación, pero el caso tiene unos límites y unas características, de allí que el investigador sea capaz de dar coherenciay secuencia a la investigación.

Simons (2011), asume que el investigador en el estudio de casos, es el principal instrumento en la recolección de datos, la interpretación y el informe, diferente de un enfoque positivista, donde el instrumento es menos falible, aquí el investigador, como instrumento, interpreta la información. Esta forma de hacer investigación trata de algo de mayor entidad que la mera observación hecha a un objeto o contexto de estudio, dice la autora:
Se trata de un riguroso examen de cómo nuestros valores y acciones configuran la recogida e interpretación de los datos, y de cómo nos afectan las personas y los acontecimientos presentes en el campo. En otras palabras, además de lo que averiguamos sobre el caso, descubrimos también cosas sobre nosotros mismos. (Simons, 2011, p. 21)

Para Merriam (1988), la característica más definitoria de la investigación con estudio de casos, se encuentra en la delimitación del objeto de estudio: el caso. El caso como se ha dicho anteriormente puede ser una unidad, programa, entidad o fenómeno con límites que son demarcados y delimitados por el investigador; pudiendo determinar lo que no se va a estudiar (Brown, 2008).

Neiman y Quaranta (2006), reconocen que los estudios de caso tienden a focalizar su mirada «en un número limitado de hechos y situaciones» (p. 218) para poder comprenderlos en su globalidad y contexto. En este sentido, el límite del estudio puede estar dado por el número de participantes, un periodo de tiempo de observación, o la instancia de un problema o preocupación.

Con respecto a los participantes, no existe en el estudio de casos un número exacto o rango de casos que deban abordarse, no hay reglas precisas para determinar el tamaño de la muestra (Patton, 1990), entre otras razones porque sus fines y naturaleza, no son los de los estudios de población que precisan de muestras representativas. Sin embargo, Eisenhardt (1989), considera que «un número entre 4 y 10 casos por lo general funciona bien» (p. 545). Con menos de 4 casos es difícil generar teoría y con más de 10 casos el volumen de información sería complejo de asumir.

\section{Características básicas y clasificaciones del estudio de caso}

El estudio de casos según Merriam (1988), posee las siguientes características: es particularista, en relación al enfoque especifico del caso, es su especificidad lo que lo hace especial; es heurístico, es capaz de ampliar la mirada del fenómeno, permite ampliar la experiencia, descubrir nuevos significados o confirmar lo que ya se sabía; es descriptivo, su descripción es muy completa, rica y literal del fenómeno objeto de estudio, y es inductivo, llega a conceptos o hipótesis a partir del examen de los datos recogidos en el contexto mismo.

Bonache (1999), determina unas características de los estudios de casos, en contraste con la metodología cuantitativa, al respecto afirma que, los estudios de caso no separan el fenómeno de su contexto, tienen una visión holística, parten de un marco teórico menos elaborado que se va construyendo, la elección del caso tiene un carácter teórico y no estadístico.

En cuanto a las estrategias de recolección y análisis de información, algunos autores afirman que, es posible utilizar estrategias tanto cuantitativas como cualitativas (observación, entrevista, cuestionarios, encuesta, análisis documental, etc.) (Pérez, 1994; Rodríguez et al., 1999), teniendo presente que el propósito no es generalizar los resultados, sino enriquecer el análisis y las fuentes de información.

Para Merriam (1988) y Yin (1984), los estudios de caso se pueden clasificar según la naturaleza del informe en descriptivos, interpretativos o evaluativos. Descriptivos: presenta un informe minucioso del caso eminentemente descriptivo, no se guían por generalizaciones, fundamentaciones teóricas, ni hipótesis previas. Son útiles para aportar información básica en ciertas áreas educativas; en educación suelen usarse en programas y prácticas innovadoras. Interpretativo: aporta descripciones densas y ricas con el propósito de interpretar y teorizar sobre el caso de estudio. El modelo de análisis es inductivo para desarrollar categorías conceptuales que ilustren, ratifiquen o desafíen presupuestos teóricos difundidos antes de la obtención de la información. Evaluativos: envuelve una descripción, explicación y juicio del caso para tomar decisiones, son útiles para la evaluación educativa, por su capacidad para explicar los vínculos causales de las intervenciones en la vida real, se usa este caso para descubrir el contexto real en el que se ha realizado la intervención y para explorar determinadas situaciones donde no se han alcanzado resultados claros.

Los estudios de caso, también pueden clasificarse según el objetivo 
fundamental que se persigue en el estudio. Según Stake (2010), se presentan las siguientes modalidades: intrínseco de casos, instrumental de casos y estudio colectivo de casos. Intrínseco: cuando el caso se estudia por su propio interés intrínseco; el propósito es alcanzar una mayor comprensión del caso en sí mismo. Instrumental: cuando el caso se elige para estudiar un tema o una pregunta de la investigación determinada de otros ámbitos, es decir, el caso se escoge para conseguir entender otra cosa; el caso es un instrumento para conseguir otros fines indagatorios. Colectivo: se usa para estudiar varios casos y hacer una interpretación colectiva del tema o la pregunta de estudio.

En relación a las unidades de análisis del estudio de caso, se ha clasificado en caso único, este solo toma una unidad de análisis y mantienen el carácter holístico o general del mismo. Caso único con subunidades de análisis dentro del mismo caso general. Estudios multicaso, cuando existen varios casos; y los multicasos con subunidades, son varios casos con diferentes subunidades de análisis (Baxter \& Jack, 2008; Neiman \& Quaranta, 2006; Yin, 1984).

Según Stake (2010), cuando se opta por un estudio de caso colectivo, debe estudiarse cada caso en profundidad para aprender acerca de su complejidad y singularidad. Una vez analizados individualmente se deben estudiar las convergencias y divergencias entre los casos (Tójar \& Mena, 2011) y así llegar a formular afirmaciones sobre el objeto de estudio.

En la figura 1, se pueden observar algunos de los aspectos mencionados sobre las clasificaciones de los estudios de caso.

\section{Principales críticas al estudio de caso}

Aun reconociendo las bondades de este enfoque investigativo, es importante reconocer que su alcance es limitado (Yin, 1984). Las mayores críticas a este método de investigación resaltan la marcada subjetividad que lleva consigo (Álvarez \& San Fabián, 2012; Biddle, 1989; Simons, 2011), el poco rigor metodológico en términos científicos positivos (Flyvbjerg, 2006) y especialmente la escasa capacidad para generalizar sus resultados (Biddle \& Anderson, 1989; Brown, 2008; Flyvbjerg, 2006; Martínez, 2006; R. Sánchez, 2013).

Sin embargo, Alves-Mazzotti (2006) recuerda que el objetivo principal del estudio de caso no es generalizar, pero a partir de los resultados sí se pueden generar proposiciones teóricas que serían aplicables a otros contextos. En palabras de Stake(2010), se puede llegar a «generalizaciones menores» (p. 20), pero el finúltimo del estudio de caso es comprender y entender su esencia, no aplicar los resultados a todos los casos

\begin{tabular}{|c|c|}
\hline $\begin{array}{l}\text { Tipos de estudio } \\
\text { de caso }\end{array}$ & Definición \\
\hline Explicativo & $\begin{array}{l}\text { Este tipo de estudio de caso sería usado si usted estuviera buscando } \\
\text { responder una pregunta que desea explicar las relaciones causales asumidas } \\
\text { en intervenciones en la vida real que son demasiado complejas para un } \\
\text { estudio o estrategias experimentales. En el lenguaje de la evaluación, las } \\
\text { explicaciones vincularían la ejecución del programa con los efectos del } \\
\text { programa (Yin, 2003) }\end{array}$ \\
\hline Exploratorio & $\begin{array}{l}\text { Este tipo de estudio de caso es utilizado para explorar aquellas situaciones en } \\
\text { las cuales la intervención que está siendo evaluada no tiene claridad, un } \\
\text { conjunto de resultados. (Yin, 2003). }\end{array}$ \\
\hline Descriptivo & $\begin{array}{l}\text { Este tipo de estudio de caso es utilizado para describir una intervención o } \\
\text { fenómeno y el contexto de la vida real en la cual ocurre (Yin, 2003) }\end{array}$ \\
\hline $\begin{array}{l}\text { Estudio de caso } \\
\text { múltiple }\end{array}$ & $\begin{array}{l}\text { Un estudio de caso múltiple permite al investigador explorar las diferencias } \\
\text { dentro y entre los casos. El objetivo es replicar los hallazgos a través de los } \\
\text { casos. Debido a que se harán comparaciones, es imperativo que los casos se } \\
\text { elijan cuidadosamente para que el investigador pueda predecir resultados } \\
\text { similares a través de los casos, o predecir resultados contrastantes sobre la } \\
\text { base de una teoría (Yin, 2003). }\end{array}$ \\
\hline Intrínseco & $\begin{array}{l}\text { Stake (1998) utiliza el término intrínseco y sugiere que los investigadores } \\
\text { que tienen un interés genuino en el caso deberían usar este método cuando la } \\
\text { intención es comprender mejor el caso. No se lleva a cabo principalmente } \\
\text { porque el caso representa otros casos o porque ilustra un rasgo o problema } \\
\text { particular, pero debido a que es particular y ordinario, el caso en sí mismo es } \\
\text { de interés. El objetivo NO es llegar a entender algún constructo abstracto o } \\
\text { un fenómeno genérico. El propósito NO es construir teoría (aunque es una } \\
\text { opción; Stake, 1998). }\end{array}$ \\
\hline Instrumental & $\begin{array}{l}\text { Se utiliza para llevar a cabo algo más que la comprensión de una situación } \\
\text { particular. Se ofrece información sobre un asunto o ayuda a refinar la teoría. } \\
\text { El caso es de interés secundario, este juega un papel de apoyo, facilitando la } \\
\text { comprensión de otra cosa. El caso es a menudo visto en profundidad, sus } \\
\text { contextos examinados, sus actividades ordinarias detalladas, y por esto } \\
\text { ayuda al investigador a perseguir el interés externo. El caso puede o no } \\
\text { puede ser visto como típico de otros casos (Stake, 1998). }\end{array}$ \\
\hline Colectivo & $\begin{array}{l}\text { Los estudios de casos colectivos son similares en naturaleza y descripción de } \\
\text { los estudios de caso múltiple (Yin, 2003) }\end{array}$ \\
\hline
\end{tabular}

(Yacuzzi, 2005). En palabras de Álvarez y San Fabián (2012), es más oportuno hablar de «transferencia» y no de «generalización» dado el paradigma cualitativo en el cual se enmarca.

Estas críticas están ligadas a una idea reduccionista de ciencia, arraigadas al paradigma positivista, sin embargo, el estudio de caso constituye un método válido para investigar en ciencias sociales como lo afirma Flyvbjerg (2006), pero debe ampararse en los criterios de rigor y credibilidad que garanticen la legitimidad del conocimiento.

\section{Criterios y estrategias de rigor en el estudio de caso}

Los conceptos de fiabilidad, objetividad y validez tienen un significado diferente en la investigación cualitativa al otorgado en el enfoque positivista (Goetz \& LeCompte, 1988; Rodríguez et al., 1999). Desde el punto de vista metodológico, la legitimación del conocimiento construido en la investigación cualitativa se fundamenta en el diálogo y la intersubjetividad.

En consecuencia, el estudio de caso debe basarse en criterios de rigor y credibilidad para validar sus hallazgos y conclusiones. Lincoln y Guba (1985) y Guba (1989), desarrollaron un sistema alternativo para valorar el rigor de la investigación naturalista que no estuviera anclada a términos racionalista. Estos autores consideran que, el rigor científico debe erigirse sobre cuatro aspectos: valor de verdad, aplicabilidad, consistencia y neutralidad. Para una mejor comprensión se realiza la similitud entre los términos racionalistas y sus equivalentes en el paradigma naturalista (figura 2).

En definitiva, para garantizar la credibilidad de los resultados emitidos por los estudios de caso, es imprescindible que se realice una descripción detallada sobre cómo fue recogida y analizada la información, en qué contexto se realiza la investigación y cómo se llevó a cabo el análisis, minimizando así los riesgos de emitir conclusiones sin soportes teóricos o empíricos.

\begin{tabular}{ccc}
\hline Aspecto & Término científico & Término naturalista \\
\hline Valor de verdad & Validez interna & Credibilidad \\
\hline Aplicabilidad & $\begin{array}{c}\text { Validez externa } \\
\text { Generalizabilidad }\end{array}$ & Transferibilidad \\
\hline Consistencia & Fiabilidad & Dependencia \\
\hline Neutralidad & Objetividad & Confirmabilidad \\
\hline
\end{tabular}

Figura 2. Términos racionalistas y naturalistas, apropiados para los cuatro aspectos de credibilidad (Guba, 1989, p. 153).

\section{Metodología}

Esta revisión teórica incluyó procesos propios de la investigación documental, asumida por Galeano (2004b) como «una privilegiada técnica para rastrear, ubicar, inventariar, seleccionar y consultar las fuentes y los documentos que se van a utilizar como materia prima de una investigación» (p.120). Asimismo, se llevó a cabo un análisis cualitativo sobre algunas investigaciones de la enseñanza que han utilizado el estudio de caso.

El rastreo se centró en dos tipos de búsquedas; por un lado, textos de investigación cualitativa que hicieran alusión a los estudios de casos (37 en total). A partir de ellos, se busca ilustrar las definiciones, clasificaciones, características y particularidades de los estudios de caso como diseño de investigación cualitativa, y a la vez, hacer una lectura de ellos, buscando la relevancia para los procesos de indagación en el campo de la educación física, el deporte y la actividad física. En segunda instancia, se tomaron investigaciones de caso en el área, publicadas en los últimos 10 años en artículos científicos o tesis doctorales (23 estudios en total).

Los casos que se presentan son ilustrativos y dan cuenta de dos categorías o intensiones básicas, por un lado, mostrar sobre qué tipo de actor o caso se hace la investigación (estudiantado, profesorado o institución); y por el otro, ilustrar los múltiples instrumentos que son susceptibles de usar en este tipo de investigación, presentando el estudio de caso como una herramienta de indagación flexible y adaptable a las situaciones que se presentan en el estudio de las realidades y actores del campo. Las fuentes analizadas, fueron sintetizadas en Resúmenes Analíticos de Investigación (RAI), que posteriormente fueron categorizadas y analizadas utilizando una hoja de cálculo en Excel (versión 2014). 


\section{Aplicaciones prácticas}

Parte de los resultados de este trabajo, se plasman en la introducción de este artículo, donde se presentan algunas definiciones, clasificaciones, características y particularidades de los estudios de caso, teniendo como referencia los principales autores usados por diferentes investigaciones en el ámbito de la educación física, lo queno significa que sean de uso exclusivo de la educación física. A continuación, se presentan de manera ilustrativa estudios que, desde la educación física, el deporte y la actividad física han utilizado el estudio de caso como estrategia cualitativa, atendiendo a dos criterios fundamentales: los actores principales en los cuales está basado el estudio (estudiantado, profesorado e instituciones) y, las principales técnicas e instrumentos utilizados para acceder a la información.

\section{Estudios donde prima la voz del profesorado}

En esta clasificación, se quiere resaltar cómo el estudio de caso se ha empleado para conocer percepciones, creencias y apreciaciones de los profesores sobre sus inquietudes y prácticas en diferentes contextos del campo, se destacan los siguientes:

Arribas (2008), tuvo por objeto conocer y valorar las creencias y teorías implícitas de seis profesores universitarios responsables de las asignaturas de actividad física en el medio natural en España. Utilizó entrevistas, historias de vida y análisis documental.

Azevedo (2009), pretendió comprender la percepción de los profesores en relación a la organización y sistematización de los contenidos de educación física en la etapa inicial en Brasil (1 a 5 años). Participaron 12 maestros de educación física de tres colegios de la región del sur de Brasil. Utilizó entrevistas semiestructuradas, cuestionarios para la caracterización de las escuelas y cuestionario para la caracterización de los profesores participantes.

Pradas (2010), analizó el pensamiento de una profesora de educación física en relación a su toma de decisiones interactivas. Para la recolección de datos utilizó grabación en vídeo de seis sesiones de clase y micrófonos inalámbricos a la profesora y cuatro estudiantes, así como entrevistas de autoconfrontación y análisis de documentos.

Díaz del Cueto, Hernández-Álvarez y Castejón (2012) analizaron las preocupaciones e intervenciones de cinco profesores en la utilización de la estrategia preguntas-respuestas durante la implementación de un cambio en enseñanza deportiva bajo el modelo Teaching Games for Understanding (TGfU). Para ello utilizaron el diario del profesor, reuniones del grupo profesores-investigadores y grabaciones de clases.

Jinhong (2012), investigó la naturaleza de la reflexión de profesores de educación física con experiencia, en cuanto a su enfoque y papel. En el estudio participaron 3 docentes de educación física expertos a través de un estudio de caso múltiple. Utilizó entrevistas semiestructuradas, observaciones no participantes, reflexiones escritas de los profesores, muestras de trabajo de los estudiantes y notas de campo del investigador.

Velázquez, Fraile y López (2014), se centraron en conocer y analizar la concepción que tienen sobre el aprendizaje cooperativo (AC) siete docentes de educación física de diferentes niveles educativos (primaria, secundaria y universidad) que son reconocidos como referentes en esta metodología. Los instrumentos fueron entrevistas, observación no participante y análisis documental.

Hortigüela, Pérez y Fernández (2016) tenían por objetivo conocer cómo se aborda la educación física en la formación inicial del profesorado en España y Costa Rica. Participaron 81 estudiantes (futuros profesores), 43 de España y 37 de Costa Rica, y dos de sus profesores. Los instrumentos utilizados fueron: análisis de guías docentes, grupos de discusión y entrevistas.

Flores, Prat y Soler (2017), analizaron las percepciones del profesorado de Educación Física sobre los efectos y consecuencias que ha generado la presencia de estudiantes de origen extranjero en su labor profesional y en la dinámica de clase. Para ello se ha realizado un estudio de casos múltiple con doce docentes de escuelas públicas multiculturales con más del 50\% de alumnado de origen extranjero. Los instrumentos utilizados para la obtención de la información fueron las entrevistas semiestructuradas y las observaciones no participantes.

Chaverra (2017), el estudio pretendió conocer e interpretar el discurso evaluativo de seis docentes de educación física durante la fase interactiva. Se utilizó el estudio de caso múltiple, se utilizaron grabaciones en video y audio de todas las sesiones de clase de una unidad didáctica y se utilizó un instrumento para la transcripción y análisis del discurso.

\section{Estudios donde prima la voz del estudiantado}

Dado el carácter dialógico de los procesos educativos (enseñanzaaprendizaje), es comprensible que, en el proceso de búsqueda de casos en educación física, aparezcan estudios ilustrativos donde se privilegie la voz del estudiantado, en los cuales se pudo evidenciar, voces referidas a aprendizajes derivados del área, y sobre todo a relaciones entre sus pares y los profesores, tal como se aprecia en los siguientes estudios:

Sánchez (2009), en un estudio de caso comparativo, se propuso observar, analizar e interpretar el clima de aula de las clases en cuatro grupos de educación secundaria obligatoria, para ello utilizó la observación, registros anecdóticos, diarios de campo, entrevistas, cuestionarios y sociogramas.

With-Nielsen y Pfister (2011), desarrollaron un estudio sobre los discursos de género en la clase de educación física en una escuela de enseñanza secundaria de Dinamarca. Utilizaron entrevistas a profundidad y observaciones de video.

Gaviria y Castejón (2013), analizaron las actitudes y valores que generan las clases de educación física en 81 estudiantes de secundaria de un instituto de la comunidad autónoma de Madrid, España; para ello se valieron de la observación y el grupo focal.

Chicon, Huber, Albiás, Sá y Estevão (2016) se propusieron describir y analizar la acción mediadora de los profesores de Educación Física en el proceso de interacción de alumnos con y sin deficiencias en una ludoteca. Los participantes fueron quince niños sin deficiencias y cinco con deficiencias. Como instrumentos utilizaron diario de campo, fotografías y video de las clases.

Gaviria y Castejón (2016b), identificaron y analizaron las creencias y percepciones de 103 estudiantes de secundaria sobre la asignatura de educación física, para ello utilizaron el cuestionario, la observación y el grupo focal.

Gaviria y Castejón (2016a), tuvieron como objetivo comprender las actitudes y valores que genera la clase de educación física en 81 estudiantes de secundaria; utilizaron la observación y el grupo focal.

Zapata, Calderón y Gaviria (2018), pretendieron comprender las características que debe tener un buen profesor desde la voz de los estudiantes de educación secundaria. Los participantes fueron 95 estudiantes de grado once y dos profesores seleccionados por los estudiantes como los mejores en su proceso educativo. Para la recolección de información se utilizó el cuestionario y la entrevista semiestructurada.

\section{Estudios sobre realidades institucionales y prácticas educati-} vas

En el proceso de codificación y análisis, también emergieron estudios donde el interés fue dar cuenta de asuntos direccionados desde las instituciones donde tienen génesis las intenciones educativas, o se indaga por una práctica educativa o estrategia en particular, lo que denota, por un lado, que un caso no siempre se centran en un sujeto o grupo de ellos (aunque casi siempre se acude a ellos como fuente de información); y por el otro, que la dinámica de la educación física, también obedece a asuntos contextuales y propósitos formativos, a continuación se hace alusión a algunos de esos casos:

Arboleda y Gaviria (2009) quisieron comprender cuáles eran las dinámicas relacionadas con la convivencia que ha generado un programa deportivo en una comunidad específica de la ciudad de Medellín-Colombia; para ello utilizaron entrevistas semiestructuradas, observacio- 
nes y talleres de grupo.

López-Pastor, Castejón y Pérez-Pueyo (2012), promovieron una calificación de exámenes como estrategia de aprendizaje en tres universidades. Tras la realización del examen, se realizó una actividad de coevaluación corrigiendo el examen in-situy luego, el alumnado diligenció un cuestionario en el que valoró esa experiencia de coevaluación. A partir de esta experiencia los objetivos de la investigación fueron: (a) comparar la calificación otorgada por los iguales con la otorgada por el profesorado; y (b) obtener las valoraciones del alumnado sobre la experiencia. Los instrumentos fueron el examen específico de la asignatura, la plantilla de corrección para cada examen y el cuestionario anónimo.

Pill, Penney y Swabey (2012), exploraron la reflexión sobre la forma y los contenidos de la enseñanza de los deportes en la clase de educación física en cincuenta y dos educadores físicos en formación. Utilizaron encuestas y entrevistas semiestructuradas.

Sánchez (2013) pretendió desarrollar un proyecto curricular de enseñanza de los juegos deportivos, con un enfoque dirigido a la comprensión táctica con el alumnado de educación secundaria, para ello se valió de la observación, la entrevista, el diario del profesor, las grabaciones y fichas elaboradas por los propios estudiantes.

Martos-García y otros (2014), analizaron las opiniones del profesorado y el alumnado frente a una programación de aula afín a la pedagogía crítica y, diseñada bajo dos principios de procedimiento: el fomento de la autonomía y de la responsabilidad, utilizando como técnicas de recogida de información la observación, entrevistas grupales y las actas de reunión de los profesores.

Bianchi y Pires (2015), indagaron la inserción de las a TICs en la formación de profesores en tres universidades de Brasil, para ello se basaron en el análisis documental, las entrevistas y las observaciones.

Merino, Arraiz y Sabirón (2017) querían conocer la adherencia de las familias en el escenario del fútbol en niños de la categoría prebenjamín. Los participantes fueron 12 jugadores, 3 entrenadores y los familiares de los niños. Se valieron de la observación participante, el análisis de fotografías y tres plataformas digitales como fuentes de datos: YouTube, un blog digital y la aplicación móvil WhatsApp.

\section{Conclusiones}

El estudio de caso como alternativa metodológica en el campo de la educación física, el deporte y la actividad física, reconoce las prácticas del área y rescata el mundo de la interioridad de los sujetos con el contexto y con los otros actores del proceso educativo. Reconoce en las diferentes prácticas, las percepciones, creencias, emociones, vivencias, opiniones, visiones y significados que poseen las personas sobre la educación física, el deporte, la recreación, la actividad física, la expresión corporal, la salud, etc. Siendo un propósito del estudio de caso en el campo, comprender la realidad social desde dentro, captar el sentido de lo que las personas quieren decir, sus silencios, sus acciones y así, entender aspectos comunes a varias personas, programas, instituciones y comunidades.

La investigación por medio de estudio de caso, da la posibilidad de comprender las conductas de las personas en la clase, el entrenamiento, los eventos deportivos, recreativos y culturales, de reconocer las interacciones entre estudiantes, deportistas, usuarios de programas, profesores, etc.; lo cual puede facilitar la puesta en práctica de mejoras personales, profesionales, institucionales o creación de nuevas orientaciones o normas sociales. El estudio de caso, da la posibilidad que el profesor e investigador, se desprenda o termine despojándose de las nociones y teorías preconcebidas, mejorando su práctica y aprendizaje como docente e investigador. En este sentido, el uso y desarrollo práctico de esta metodología es de gran utilidad para el área.

Las investigaciones reseñadas en los párrafos anteriores, se llevaron a cabo en diversos ámbitos de la educación física, el deporte y la actividad física, en todos los niveles educativos y con diferente población, objetivos y técnicas; lo cual genera un amplio conocimiento en el área y, a la vez, refleja la versatilidad del estudio de caso como herramienta.

Frente a la utilización de técnicas e instrumentos, vemos como los estudios de caso no abogan por alguna o algunas estrategias en particu- lar, todo depende del tipo de caso y de la situación a estudiar. Sin embargo, se fundamentan en las apreciaciones de los sujetos y las circunstancias sociales que se derivan de sus contextos, tal como lo consideran López, Monjas y Pérez (2003). Desde esta perspectiva, se reconoce el estudio de caso como una estrategia fundamental para el desarrollo científico del área, ya sea como método en sí mismo, o como complemento o alternativa de otros métodos de investigación.

Finalmente, reconocemos que la investigación en la educación física, el deporte y la actividad física, requiere avanzar en el sentido de comprender y conocer lo que el estudiantado, el profesorado y demás actores del área, piensan, sienten, creen y perciben sobre el campo. Las creencias y percepciones pueden ser una fuente inestimable de información y aprendizajes para el desarrollo y mejora de la educación física. Como plantea Ruiz (2011), toda actividad humana y lo que sucede en el contexto escolar es susceptible de mejora y de transformaciones, sin embargo, no siempre se hace con la inteligencia necesaria, para que todos puedan disfrutar de las bondades que estos espacios o actividades nos pueden brindar, de allí que presentemos el estudio de caso como una alternativa que permita acceder a este conocimiento.

\section{Referencias}

Álvarez, C., \& San Fabián, J. (2012). La elección del estudio de caso en investigación educativa. Gazeta de Antropología, 28(1).

Alves-Mazzotti, A. (2006). Usos e abusos dos estudos de caso. Cadernos de pesquisa, 36(129), 637-651.

Arboleda, V., \& Gaviria, D. (2009). El conflicto: aprendizaje para la convivencia o escenario para ejercer el poder de excluir como castigo: el caso de la Escuela Popular del Deporte en el corregimiento de San Cristóbal, Medellín, 2007-2008. Educación Física y Deporte, 28(1), 29-38.

Arribas, H. (2008). El pensamiento y la biografiì del profesorado de Actividad Fìsica en el Medio Natural: un estudio multicaso en la formacioin universitaria orientado a la comprensioìn de modelos formativos. (Tesis Doctoral), Universidad de Valladolid, Valladolid.

Azevedo, E. (2009). Percepções dos professores de educação física sobre as orientações curriculares: estudo de caso dos colégios de aplicação no sul do Brasil. (Tesis Doctoral), Universidade do Minho, Braga.

Baxter, P., \& Jack, S. (2008). Qualitative Case Study Methodology: Study Design and Implementation for Novice Researchers. The Qualitative Report, 13(4), 544-559.

Bianchi, P., \& Pires, G (2015). Cultura digital e formação de professores de educação física: Estudo de caso na Unipampa. Movimento, 21(4), 1025-1036.

Biddle, B., \& Anderson, D. (1989). Teoría, métodos, conocimientos e investigaciones sobre la enseñanza. En M. Wittrock (Ed.), La investigación de la enseñanza I (pp. 93-148). Barcelona: Paidós.

Bonache, J. (1999). El estudio de casos como estrategia de construcción teórica: características, críticas y defensas. Cuadernos de Economía y Dirección de la Empresa, 3, 123-140.

Brown, P. (2008). A Review of the Literature on Case Study Research. Canadian Journal for New Scholars in Education, 1(1), 1-13.

Chatoupis, C., \& Vagenas, G. (2011). An analysis of published processproduct research on physical education teaching methods. International Journal of Applied Sports Sciences, 23(1), 271-289.

Chaverra-Fernández, B. (2017). Análisis del discurso evaluativo. Infancia, Educación y Aprendizaje, 3(2), 723-728. https://doi.org/http:/ /dx.doi.org/10.22370/ieya.2017.3.2.808

Chicon, J., Huber, L., Albiás, T., Sá, M., \& Estevão, A. (2016). Educaçâo física e inclusâo: A mediaçâo pedagógica do professor na brinquedoteca. Movimento, 22(1), 279-292.

Cohen, L., Manion, L., \& Morrison, K. (2007). Research methods in education (6a). New York: Routledge.

Coller, X. (2000). Estudio de casos. Madrid: Centro de Investigaciones Sociológicas.

Díaz-del-Cueto, M., Hernández-Álvarez, J., \& Castejón, F. (2012). La estrategia preguntas-respuestas como clave de la enseñanza comprensiva del deporte en Educación Física: estudio de casos. Cultura 
y Educación, 24(3), 273-288. https://doi.org/10.1174/ 113564012802845677

Eisenhardt, K. (1989). Building Theories from Case Study Research. The Academy of Management Review, 14(4), 532-550.

Flick, U. (2004). Introduccioin a la investigacioìn cualitativa. Madrid: Morata.

Flores, G, Prat, M., \& Soler, S. (2017). La visión del profesorado de Educación Física sobre la presencia del alumnado de origen extranjero en la escuela: ¿oportunidad o problema? Retos. Nuevas tendencias en Educación Fisica, Deporte y Recreación, $1^{\circ}$ semestr(31), 64-68.

Flyvbjerg, B. (2006). Five Misunderstandings About Case-Study Research. Qualitative Inquiry, 12(2), 219-245. https://doi.org/ 10.1177/1077800405284363

Galeano, M. (2004a). Diseño de proyectos en la investigación cualitativa. Medellín: Universidad Eafit.

Galeano, M. (2004b). Estrategias de investigación social cualitativa. El giro de la mirada. Medellín: La Carreta.

Gaviria, D., \& Castejón, F. (2013). El proceso didáctico en educación física como generador de valores y actitudes. Qualitative research education, 2(2). https://doi.org/10.4471/qre.2013.22

Gaviria, D., \& Castejón, F. (2016a). Desarrollo de valores y actitudes através de la clase de Educación Física. Movimento, 22(1), 251262.

Gaviria, D., \& Castejón, F. (2016b). La educación física en la voz de los estudiantes de secundaria. Un estudio de caso. Cultura, Ciencia y Deporte, 11(32), 107-118. https://doi.org/http://dx.doi.org/ 10.12800/ccd.v11i32.711

Gerring, J. (2004). What is a case study and what is it good for? American Political Science, 98(2), 341-354.

Goetz, J., \& LeCompte, M. (1988). Etnografía y diseño cualitativo en investigación educativa. Madrid: Morata.

Guba, E. (1989). Criterios de credibilidad en la investigación naturalista. En J. Gimeno \& A. Pérez (Eds.), La enseñanza, su teoría y su práctica (pp. 148-165). Madrid: Akal.

Hernández, J. (2004). Teoría curricular y didáctica de la Educación Física. En A. Fraile (Ed.), Didáctica de la Educación Física. Una perspectiva crítica y transversal (pp. 29-61). Madrid: Biblioteca nueva.

Hortigüela, D., Pérez, Á., \& Fernández, J. (2016). Entender y enseñar la educación física en la formación inicial del profesorado: Estudio de Caso en España y Costa Rica. Movimento, 22(4), 1049-1062.

Jinhong, J. (2012). The focus, role, and meaning of experienced teachers' reflection in physical education. Physical Education and Sport Pedagogy, 17(2), 157-175. https://doi.org/10.1080/ 17408989.2011.565471

López-Pastor, V., Castejón, F., \& Pérez-Pueyo, Á. (2012). ¿Implicar al alumnado en la evaluación en la formación incial del profesorado? Un estudio de caso de evaluación entre iguales de un examen. Multidisciplinary Journal of Educational Research, 2(2), 177-201. https://doi.org/10.4471/remie.2012.09

López, V., Monjas, R., \& Pérez, D. (2003). Buscando alternativas a la reforma de entender y practicar la Educación Física escolar. Barcelona: Inde.

Martínez, P. (2006). El método de estudio de caso. Estrategia metodológica de la investigación científica. Pensamiento y Gestión, 20(Ene-Jun), 165-193.

Martos-Garcia, D., Torrent-Benavent, G, Durbà-Cardo, V., Saiz-Llopis, L., Tamarit-López, E., Valencia, U. De, ... Uixó, V. (2014). El desarrollo de la autonomía y la responsabilidad en educación física: Un estudio de caso colaborativo en secundaria. Retos. Nuevas tendencias en Educación Fisica, Deporte y Recreación, 2041(26), 3-8.

Massot, I., Dorio, I., \& Sabariego, M. (2012). Estrategias de recogida de información y análisis de la información. En R. Bisquerra (Ed.), Metodología de la investigación educativa (3ª, pp. 329-366). Madrid: La Muralla.

Merino, A., Arraiz, A., \& Sabirón, F. (2017). La adherencia del entorno familiar en el fútbol prebenjamín: un estudio de caso. Revista Iberoamericana de psicologia del ejercicio y el deporte, 12(1), 139-148.

Merriam, S. (1988). Case study research in education a qualitative approach. San Francisco: Jossey-Bass.

Neiman, G., \& Quaranta, G. (2006). Los estudios de caso en la investigación sociológica. En I. Vasilachis de Gialdino(Ed.), Estrategias de investigación cualitativa (pp. 213-234). Barcelona: Gedisa.

Patton, M. (1990). Qualitative Evaluation and Research Methods. Newbury Park: Sage.

Pérez, G. (1994). Investigación cualitativa. Retos e interrogantes II. Técnicas y análisis de datos. Madrid: La Muralla.

Pérez, G. (1999). Reflexiones sobre la investigacioìn en educacioìn social y animacioìn sociocultural. En G. Pérez (Ed.), Modelos de investigacioìn cualitativa en educacioìn social y animacioìn sociocultural (pp. 21-56). Madrid: Narcea.

Pérez, G. (2008). Investigación cualitativa. Retos e interrogantes I. Métodos (5). Madrid: La Muralla.

Pill, S., Penney, D., \& Swabey, K. (2012). Rethinking Sport Teaching in Physical Education: ACase Study of Research Based Innovation in Teacher Education. Australian Journal of Teacher Education,37(8), 118-138. https://doi.org/http://dx.doi.org/10.14221/ ajte.2012v37n8.2

Pradas, R. (2010). El estudio del pensamiento del profesorado sobre la toma de decisiones interactivas: análisis de un caso en educación física escolar. Cultura y Educación, 22(1), 21-36. https://doi.org/ 10.1174/113564010790935187

Rodríguez, G., Gil, J., \& García, E. (1999). Metodología de la investigación cualitativa $\left(2^{\mathrm{a}}\right)$. Málaga:Aljibe.

Ruiz, L. (2011). Lo que los deportes enseñan cuando se aprenden los deportes. Tándem: Didáctica de la Educación Física, 36, 17-26.

Sánchez, J. (2009). Análisis del clima del aula en educación física. Un estudio de casos. (Tesis doctoral). Universidad de Málaga, Málaga.

Sánchez, R. (2013). La enseñanza para la comprensión de los juegos deportivos: Un estudio de casos en la enseñanza secundaria. (Tesis Doctoral), Universidad de Valencia, Valencia.

Silverman, S. (1987). Trends and analysis of research on teaching in doctoral programs. Journal of teaching in physical education, 7(1), 67-70.

Silverman, S., \& Mason, M. (2003). Research on teaching in physical education doctoral dissertations: a detailed investigation of focus, method, and analysis. Journal of teaching in physical education, 22(3), 280-297.

Simons, H. (2011). El estudio de caso: teoría y práctica. Madrid: Morata. Stake, R. (2010). Investigación con estudio de casos (5a). Madrid: Morata.

Strauss, A., \& Corbin, J. (2002). Bases de la investigación cualitativa: Técnicas y procedimientos para desarrollar la teoría fundamentada. Medellín: Facultad de Enfermería de la Universidad de Antioquia.

Taylor, S., \& Bogdan, R. (1987). Introducción a los métodos cualitativos de investigación. Barcelona: Paidós.

Tójar, J., \& Mena, E. (2011). Innovaciones educativas en el contexto andaluz. Anailisis multicaso de experiencias en Educacioìn Infantil y en Educacioìn Primaria. Revista de Educación, 354(Enero-Abril), 499-527.

Velázquez, C., Fraile, A., \& López-Pastor, V. (2014). Aprendizaje cooperativo en Educación Física. Movimento, 20(1), 239-259.

With-Nielsen, N., \& Pfister, G. (2011). Gender constructions and negotiations in physical education: case studies. Sport, Education and Society, 15(5), 645-664.

Yacuzzi, E. (2005). El estudio de caso como metodologiìa de investigacioìn: teoriìa, mecanismos causales, validacioìn. Buenos Aires: Universidad del CEMA.

Yin, R. (1984). Case study research: design and methods, applied social research methods series. Newbury Park: Sage.

Zapata, J., Calderón, A., \& Gaviria, D. (2018). ¿Quién es un buen profesor? Un caso con estudiantes de educación secundaria en Medellín-Colombia. Viref Revista de Educación Física, 7(1), 1933.

$-427-$ 